\title{
Nanoscience and nanotechnology in the twenty-first century
}

\author{
Harold Kroto
}

Published online: 2 May 2013

(C) Controlled Release Society 2013

As the twenty-first century unfolds, chemistry-based, bottom-up approaches to the creation of materials with exactly specified atomic and molecular infrastructures have become more and more feasible and the top-down approach, which has served us so well up to now, appears to be approaching some fundamental size-related limits. The aim has been to achieve the promise of the creation of new materials with advanced function, e.g. highly improved tensile strengths and/or novel electronic and magnetic properties, intelligent nanoscale machines for medical applications, etc. As these new approaches to research in materials science have shifted more and more towards this more intrinsic chemical perspective, the field has acquired a new name: Nanoscience and Nanotechnology $(\mathrm{N} \& \mathrm{~N})$. It is of course not new as biology has done it this way since the first organisms appeared as all living systems are created atom-by-atom and molecule-by-molecule on the basis of a chemically coded recipe stored in DNA.

Science was born out of curiosity, not out of expedience, and it is still true today that many major breakthroughs are made by the openly curious who generally uncover breakthroughs which those with more focused attitudes tend to overlook. With the development of radio telescopes during the last half of the twentieth century, the very cold interstellar medium was found to be a veritable Pandora's Box, full to the brim with dust particles and fascinating exotic molecules as well as some highly puzzling material responsible for a plethora of as yet unidentified optical features. Particularly fascinating, curious and crucial has been the role that the element carbon has played in almost every aspect of the development of our understanding of both the physical and natural sciences. A surprise that the element had up its sleeve was the existence of $\mathrm{C}_{60}$, Buckminsterfullerene, the

H. Kroto $(\bowtie)$

Chemistry and Biochemistry Department,

The Florida State University, Tallahassee, FL, USA

e-mail: m.kroto@btinternet.com third well-defined form of carbon - the other two being graphite and diamond. Follow-up work from the $\mathrm{C}_{60}$ discovery also led to the re-discovery of the carbon nanotubes which promise paradigm shifting advances in materials engineering at nanoscale dimensions. So, curiosity about the chemistry that occurs in exameter-sized objects such as Giant Molecular Clouds in space some with diameters as large as 100 ly $\left(\sim 10^{18} \mathrm{~m}^{*}\right)$ led to the discovery of an object ca $10^{27}$ times smaller and which has become an iconic symbol of nanotechnology, i.e. the science of structures at nanoscale (ca $\left.10^{-9} \mathrm{~m}\right)$ dimensions.

Even though the $\mathrm{C}_{60}$ molecule was discovered over 25 years ago and has led to some 20,000 papers, there are still puzzling problems in understanding how it actually forms. A research collaboration at Florida State University (FSU) with Alan Marshall, using the exceptional resolution afforded by the FT-ICR-MS technology developed at the NHFML at FSU, has uncovered another piece of the jigsaw puzzle. The programme has also uncovered some fascinating and intriguing results on small fullerene cages, in particular $\mathrm{C}_{28}$. Further studies of nanoscale-structured materials at FSU in collaboration with Naresh Dalal's group have produced metal organic frameworks exhibiting fascinating dynamic behaviour.

Nanoscale molecules and related structures have properties that should be able to fulfil some of the exciting promise of twenty-first century materials science and technology. The structures, which have also become the iconic images of $\mathrm{N} \& \mathrm{~N}$, are now the subject of intense study as they promise to play key roles in almost every possible area of future technology, from medicine and molecular electronics to civil engineering. In addition to carbon-based structures, the nanoscale behaviour of numerous other related materials is being explored with similarly exciting prospects. Ingenious strategies for the creation of molecules with complex, exactly specified structures and function are being developed - basically molecules that "do something" are now being made. As an example, the drug industry has so far focused on the production of 
"relatively" simple molecules with exactly specified rigid structures but a plethora of complex molecules from electric motor enzymes and pulsing molecular machines abound within the body. These molecules are now highlighting incredibly exciting new directions for molecular biological research and challenging new perspectives for pharmaceutical research and development.

The fact that the third form of carbon had been hiding in the shadowy corners of the universe since time immemorial brings to mind the mysterious character lurking in the dark streets of Vienna, made famous by Orson Welles in the classic movie "The Third Man". Especially compelling support for the idea that $\mathrm{C}_{60}$ existed in space lay in the fact that the original discovery was made serendipitously during laboratory experiments designed to simulate the atmospheric conditions in cool red giant carbon stars. This is yet another example of the remarkable way in which the fascination with space has catalysed fundamental breakthroughs in general science with major implications for innovative technological applications on Earth. There is food for thought in the fact that $\mathrm{C}_{60}$ is now being made commercially by a relatively simple combustion technique, and yet when it was first proposed that it might play some role in combustion, fierce antagonism to this proposal came from some members of the combustion research community.

In fact, the cross-disciplinary field of $\mathrm{N} \& \mathrm{~N}$ has resulted from a deep understanding and expert application of the fundamental chemical principles that underlie condensed matter physics, molecular biology and materials engineering. Indeed, N\&N may be considered "Frontier Chemistry of the 21st Century". However, the mechanisms whereby various types of nanostructures assemble are still poorly understood. Over the last decade or so, we have examined a wide range of methods for nanostructure formation, and from these studies, important new insights have been gained. We have focused particular attention on the factors governing the creation of materials with intrinsically 1 - and 2-D nanoscale infrastructures. Although some improvement in materials behaviour has already been achieved, it is unlikely that the technological paradigm shifts that N\&N promise will be forthcoming until key fundamental mechanistic issues can be resolved and we have significantly improved fine control over bottom-up (chemical) selfassembly.

The history of scientific progress carries a serious health warning for those who think that fundamental science can be steered by bureaucratic decision making and the story of the discovery of the third form a carbon and its key role in the birth of Nanoscience and Nanotechnology is yet another salutary example.

$$
1 \text { lightyear }=9.4605284 \times 10^{15} \text { meters }
$$

deca- hecto- kilo- mega- giga- tera- peta- exa- zetta- yotta-

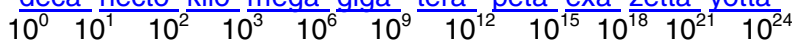
$\frac{\text { deci- }}{10^{0}} \frac{\text { centi- milli- micro- nano- pico- femto- }}{10^{-1}} \frac{\text { atto- zepto- }}{-\frac{1}{-2} 10^{-3}} 10^{-6} \frac{\text { yocto- }}{10^{-9}}$ 